\title{
BMJ Open Morita Therapy for depression (Morita Trial): an embedded qualitative study of acceptability
}

\author{
Holly Victoria Rose Sugg, ${ }^{\circledR}$ Julia Frost, ${ }^{\circledR}$ David A Richards ${ }^{\odot}$
}

To cite: Sugg HVR, Frost J, Richards DA. Morita Therapy for depression (Morita Trial): an embedded qualitative study of acceptability. BMJ Open 2019;9:e023873. doi:10.1136/ bmjopen-2018-023873

- Prepublication history and additional material for this paper are available online. To view these files, please visit the journal online (http://dx.doi. org/10.1136/bmjopen-2018023873).

Received 27 April 2018 Revised 23 October 2018 Accepted 21 March 2019
Check for updates

(C) Author(s) (or their employer(s)) 2019. Re-use permitted under CC BY-NC. No commercial re-use. See rights and permissions. Published by BMJ.

Medical School, University of Exeter, Exeter, UK

Correspondence to Dr Holly Victoria Rose Sugg; h.v.s.sugg@exeter.ac.uk

\section{ABSTRACT}

Objective To explore the views of UK-based recipients of Morita Therapy (MT) on the acceptability of MT.

Design Qualitative study nested within a pilot randomised controlled trial of MT (a Japanese psychological therapy largely unknown in the UK) versus treatment as usual, using post-treatment semistructured interviews analysed with a framework approach.

Setting and participants Participants who received MT as part of the Morita Trial, recruited for the trial from General Practice record searches in Devon, UK. Data from 16 participants were purposively sampled for analysis. Results We identified five themes which, together, form a model of how different participants viewed and experienced MT. Overall, MT was perceived as acceptable by many participants who emphasised the value of the approach, often in comparison to other treatments they had tried. These participants highlighted how accepting and allowing difficulties as natural phenomena and shifting attention from symptoms to external factors had facilitated symptom reduction and a sense of empowerment. We found that how participants understood and related to the principles of MT, in light of their expectations of treatment, was significantly tied to the extent to which MT was perceived as acceptable. Our findings also highlighted the distinction between MT in principle and practice, with participants noting challenges of engaging with the process of therapy such as fear and discomfort around rest, needing sufficient support from the therapist and others, and the commitment of treatment.

Conclusions People in the UK can accept the premise of MT, and consider the approach beneficial and novel. Therefore, proceeding to a large-scale trial of MT is appropriate with minor modifications to our clinical protocol. Participants' expectations and understandings of treatment play a key role in acceptability, and future research may investigate these potential moderators of acceptability in MT.

Trial registration numberC ISRCTN17544090; Preresults.

\section{INTRODUCTION AND OBJECTIVES}

Depression and generalised anxiety disorder (GAD) are the two most common mental health disorders: lifetime prevalence is estimated at $16.2 \%$ and $5.7 \%$ for depression and GAD, respectively. ${ }^{1-3}$ Depression is the leading cause of disability worldwide and

\section{Strengths and limitations of this study}

- This is the first study to explore the views of UKbased patients about Morita Therapy (MT).

- The use of qualitative methods generated rich insights into participants' views of MT, informing the development of a model of how different participants engaged with and experienced the approach.

- Our sample size was constrained by the number of Morita Trial participants meeting our sampling criteria. However, our purposive and theoretically driven sampling framework enabled us to explore both the breadth and depth of data, and identify issues with acceptability in particular.

- The analysis may not have readily reflected how participants' views changed over time and some challenges were encountered in establishing how acceptability was shaped by other factors and/or vice versa.

is often chronic, recurrent, comorbid with other conditions and associated with a high risk of suicide. ${ }^{24-7}$ Between 2011 and 2030, the effect of depression on global economic output is predicted to be US $\$ 5.36$ trillion. ${ }^{8}$ Medication and cognitive behavioural therapy (CBT) have the strongest evidence-base for treating these conditions. ${ }^{9} 10$ However, many people are resistant to such interventions, with between one-third and half of patients not responding to treatment. ${ }^{11-20}$ Thus, there is a scope to test new potentially effective treatments.

Morita Therapy (MT) is a Japanese psychotherapy developed by Dr Shōma Morita in 1919 and informed by Zen Buddhist principles. $^{2122}$ Key components are outlined in table 1. It is a holistic approach aiming to improve everyday functioning rather than targeting specific symptoms. ${ }^{23}$ Through conceptualising unpleasant emotions as part of the natural ecology of human experience, MT seeks to re-orientate patients in the natural world and potentiate their natural healing capacity. ${ }^{21}$ Morita therapists thus help patients to move away from symptom 
Table 1 Key components of MT

\section{Components Definition}

Natural world MT conceptualises unpleasant thoughts and emotions as part of the natural ecology of the human experience. It draws on the natural world, and the place of humans within it, to emphasise that symptoms are not subject to the patient's control, and will naturally ebb and flow with time.

Acceptance and allowance of internal states/vicious cycle

All emotions and thoughts (internal states) are accepted as they are. Any attempts to control, resist, avoid or intervene in symptoms are considered to exacerbate them within a vicious cycle; therapists thus help patients to move away from symptom preoccupation and combat and towards acceptance and a focus on action-taking. Thus, the objectives of therapy are to shift attention and perspective, and move patients to a position of accepting and responding to phenomenological reality as it is, rather than controlling or 'fixing' symptoms.

\begin{tabular}{ll} 
Normalisation & $\begin{array}{l}\text { Therapists label thoughts and emotions as 'unpleasant' and 'pleasant' but not 'good' or 'bad'. They } \\
\text { emphasise that all emotions are natural, or normal, and will ebb and flow on their own so long as } \\
\text { attempts are not made to resist them. }\end{array}$ \\
$\begin{array}{l}\text { Fumon (inattention to } \\
\text { symptoms) }\end{array}$ & $\begin{array}{l}\text { Therapists, in an effort to shift patients' attention away from symptom preoccupation and combat, } \\
\text { will not focus on discussion or analysis of patients' symptoms or their causes, but will 'steer' the } \\
\text { conversation towards action-taking and the external environment. }\end{array}$ \\
\hline Diaries & $\begin{array}{l}\text { Patients complete daily diaries on which therapists provide comments which facilitate an acceptance } \\
\text { of internal states and refocus attention on action-taking and the external environment. }\end{array}$ \\
Four-phased model & $\begin{array}{l}\text { Rest and action-taking are structured within four phases: (1) rest; (2) light repetitive activities; (3) } \\
\text { more challenging activities and (4) social reintegration. The process is understood to aid experiential } \\
\text { acceptance of the natural ebb and flow of thoughts and emotions, to re-orientate patients in nature, } \\
\text { and to refocus attention from the 'self'/internal states to external reality. }\end{array}$ \\
Rest & $\begin{array}{l}\text { MT seeks to potentiate patients' natural healing capacities, in contrast to resisting and exacerbating } \\
\text { symptoms. Patients sit with their thoughts and emotions as they are, to learn how they naturally ebb } \\
\text { and flow with time if left unattended and to build a natural desire to take action. }\end{array}$ \\
Action-taking with & $\begin{array}{l}\text { Patients learn to undertake purposeful and necessary action, with or without their symptoms; action } \\
\text { which is driven by 'desire for life' rather than undertaken for the purpose of feeling differently. MT thus } \\
\text { symptoms } \\
\text { aims to improve everyday functioning in spite of symptoms, with symptoms reducing as a by-product } \\
\text { of moving from a mood-oriented to purpose-oriented and action-based lifestyle. }\end{array}$ \\
\hline
\end{tabular}

MT, Morita Therapy.

preoccupation and combat, which are considered to exacerbate symptoms and interfere with this natural recovery process. ${ }^{24}$ By helping patients to accept symptoms as natural phenomena which ebb and flow as a matter of course, MT is in sharp contrast to the focus of established Western approaches on symptom control. ${ }^{25}$ Thus, MT has potential to provide UK patients with a distinct alternative to current National Institute For Health And Clinical Excellence (NICE)-recommended treatment options.

The Morita Trial, a mixed methods feasibility study guided by a pragmatic philosophy ${ }^{26}$ and encompassing a pilot randomised controlled trial and embedded qualitative interviews, was the first trial of MT in the UK. The trial received ethical approval from the National Research Ethics Service South West-Frenchay (reference $15 / \mathrm{SW} / 0103)$. In all, 68 participants with major depressive disorder, with or without anxiety disorder(s), were recruited through General Practice record searches in Devon, UK and randomised to receive treatment as usual (TAU) or TAU plus 8-12 sessions of MT delivered by trained therapists at the University of Exeter's AccEPT clinic following the UK MT outpatient protocol developed by the study team. ${ }^{27}$ The trial established that a large-scale MT trial is feasible with minor modifications to the pilot trial protocol, and that MT shows promise in treating depression. The protocol (see online supplementary file 1) and quantitative results are reported elsewhere. ${ }^{28} 29$

Here, we report the results of the embedded qualitative study designed to address the clinical uncertainties ${ }^{30}$ associated with a large-scale trial. As the acceptability of MT in the UK was unknown, gathering data on this was essential to inform any necessary amendments to our clinical protocol and ensure that the treatment administered in any large-scale trial is acceptable. Thus, our research question was as follows: How acceptable is MT to participants?

\section{METHODS}

\section{Design}

In-depth semistructured interviews with participants who received MT within the Morita Trial, as part of a mixed methods embedded design. ${ }^{31}$ For the quantitative (reported elsewhere ${ }^{29}$ ) and qualitative components, we collected data concurrently and analysed data sequentially (with quantitative data informing our sampling of qualitative interviews for analysis: see below). 
Table 2 Topic guide

\section{Topic of discussion}

Thoughts and feelings before treatment

Can you tell me a bit about what led you to take part in this trial?

\section{Understanding/experiences of treatment}

Having now attended MT, please can you describe to me your understanding of what MT is?

Please tell me about your experiences of receiving treatment

\section{Probe areas}

- Anything about MT in particular

- Why are they involved in this trial in particular

- What did they expect from treatment

Any problems with which they particularly wanted help

- Understanding of the goals of MT

- What it felt like receiving treatment

- Anything in particular that they liked or found helpful

- Anything they did not like or found less helpful

- How could the treatment have been improved

- How well the therapy helped them with the problems they wanted to work on

- To what extent did therapy match their expectations - why (not)/how (not)/in what ways

- Length of therapy sessions/total length of treatment/way in which therapy was ended

- Each phase of therapy

- Diary and written comments

- Style of the therapist

- Concept of 'desire for life'

- Concept of the vicious cycle

- Idea of accepting the natural ebb and flow of emotions

- Connection to natural world

- Use of metaphors

- Amount of explanation and rationale provided

- Personal contextual factors

- Specific therapy factors

- Therapeutic relationship factors

- Anything (else) that could have been done to overcome these difficulties

- Anything they learnt during treatment

- What was it about treatment which brought about any changes

- What impact did such changes have for them anything you may have learnt from the treatment. Please can you tell me about any changes that happened for you during treatment?

MT, Morita Therapy.

\section{Recruitment and data collection}

At baseline, we asked participants whether they would be willing to be interviewed about their experiences of therapy. On therapy completion, we invited consenting participants allocated to MT $(n=34)$ to interview, conducted at University of Exeter premises or the participant's home, depending on participant preference.

We developed a semistructured topic guide (table 2) based on recent mental health trials addressing similar questions, ${ }^{19} 3233$ MT literature and our MT optimisation study findings. ${ }^{27}$ Topics included participants' thoughts and feelings before treatment, understandings and experiences of treatment, barriers to treatment and mechanisms of change, which were initially explored using open-ended questions defining these areas ${ }^{34}$ (table 2: topic of discussion). While our topic guide thus helped to ensure consistency in data collection and allow for comparison across participants, interviews were interactive, responsive and flexible in order to pursue unanticipated views and the most salient details for each participant ${ }^{34}$ : individual responses were probed to investigate participants' meanings, enabling both the exploration of participants' views on pre-defined topics of interest and the elicitation of participants' own themes. ${ }^{36}$ The probe areas included in our topic guide (table 2) thus served as an aide-memoire for the interviewer to ensure discussion of each element of interest, and informed follow-up questions which were asked in a flexible manner dependent on the information already provided by the interviewee. ${ }^{34} 37$ Following the first three interviews, we amended the topic guide to include probe areas based on the views already elicited.

With participants' permission, interviews were audio-recorded and transcribed verbatim. Transcripts were checked for accuracy. We made field notes during each interview and summarised these at interview completion, which helped inform topic guide amendments, facilitated sampling and were referred to alongside transcripts during analysis. 


\begin{tabular}{lllll} 
& & \multicolumn{2}{l}{ Adherence to treatment } \\
\cline { 3 - 5 } & & Withdrew $<$ 5 sessions & Withdrew $\mathbf{2 5}$ sessions & Completed treatment \\
\hline Treatment response? & Yes & $n=3$ & $n=1$ & $n=6$ \\
(follow-up PatientHealth & No & $n=2$ & $n=3$ & $n=1$ \\
Questionnaire 9 score <10) & & & & \\
\hline
\end{tabular}

\section{Sampling}

Using a nested sampling design, we analysed data from a subsample of interviews. ${ }^{38}{ }^{39}$ This approach enabled us to purposively select 'key informants' [38 p.240] on acceptability according to theoretically driven criteria deemed important in answering our research question. ${ }^{39} 40$ These criteria were as follows: (1) treatment adherence, given the potential for this to be related to views of therapy; (2) whether participants demonstrated a response to treatment (ie, Patient Health Questionnaire $9^{41}$ score $<10$ at follow-up), given the potential for participants' views to be confounded by their degree of symptom improvement. Our objective was to include a quota of three participants within each subgroup in the resulting sampling matrix (table 3 ).$^{39}$ We therefore selected participants in order to achieve maximum variation according to these criteria, and aimed to both capture the breadth of views on acceptability and explore the depth and diversity of views within each subgroup. ${ }^{35} 4042$ For participants who both completed and responded to treatment, we further purposively sampled data according to additional criteria deemed potentially relevant: presence or not of GAD at baseline; participants' experience or not of CBT; participants' gender; therapist.

In determining the sample size, we prioritised the study purpose in order to achieve sampling adequacy: we estimated the number of participants required to sufficiently answer the research question by achieving both breadth and depth of information. ${ }^{3542-45}$ This estimation was informed by the concept of data saturation ${ }^{46-48}$ and related findings ${ }^{49}$ which suggested that an analysis of 12 interviews could provide a thorough picture of participants' views. The final sample size was informed by the heterogeneity of the population, the number of selection criteria and the number of participants meeting these criteria. $^{35}$

\section{Analysis}

We analysed data in NVivo10 $0^{50}$ using Framework analysis $^{35}$ to allow for an abductive approach. We achieved familiarisation with the data through reading transcripts, and following the guidance of Miles, Huberman and Saldana ${ }^{39}$ completed first cycle coding and developed an initial thematic framework as batches of transcripts were analysed, iteratively combining the topic guide and the overall impression of the narratives in context. Using this framework, we completed second cycle coding on individual transcripts and analysed them thematically using a constant comparison approach. ${ }^{39}{ }^{51}$ We charted data in analytic/framework matrices, as per the Framework approach, ${ }^{35}$ to allow within and across case analyses and the exploration of relationships between themes; throughout this process, we interpreted data with the aid of thematic maps to make sense of participants' perspectives, understand and structure the relationships between themes, and conceptualise the overall picture of participants' views. ${ }^{35}$ 52-54 We explored negative cases and provided explanations of variance, ${ }^{55}$ ensuring all observations relevant to the research question were incorporated. Two authors (HVRS and JF) conducted data analysis, each coding raw data (HVRS all data; JF a subset) and meeting regularly to develop themes and discuss data interpretation.

\section{Patient involvement}

The Morita Trial follows on from an iterative programme of work conducted to develop our MT clinical protocol, whereby we optimised MT according to the views of potential patients and therapists. ${ }^{27}$ The patient materials were developed on the basis of consultation with a Public and Patient Involvement (PPI) expert and similar materials used in other mental health trials which had received feedback from PPI groups (eg, PenPIG http:// clahrc-peninsula.nihr.ac.uk/). A former trial participant, who expressed an interest in supporting our research and will be involved in the further dissemination of results, has co-written a summary sheet explaining our results in lay terms which has been sent to consenting former trial participants.

\section{RESULTS}

From November 2015 to January 2017, one author (HVRS) completed 28 interviews with MT participants lasting between 24 and $93 \mathrm{~min}$. We did not interview six participants because they could not be contacted $(n=3$; $8.8 \%)$; declined $(n=2 ; 5.9 \%)$ or had moved away $(n=1$; 2.9\%). We sampled 16 interviews for analysis (table 3): these included all participants who did not complete and/or did not respond to treatment $(n=10)$ and six who completed and responded to treatment. Thus, only additional participants who both completed and responded to treatment were not sampled for analysis. Participant characteristics are provided in table 4 .

Participants' views were understood within five themes: (1) the impact of incompatible expectations and understandings; (2) identifying with the principles of MT: receptivity and relevance; (3) approaching 
Table 4 Participant characteristics

\begin{tabular}{|c|c|}
\hline Characteristic & $\begin{array}{l}n(\%) \text { unless } \\
\text { otherwise stated } \\
\text { Total } n=16\end{array}$ \\
\hline \multicolumn{2}{|l|}{ Gender } \\
\hline Female & $9(56)$ \\
\hline \multicolumn{2}{|l|}{ Age (years) } \\
\hline Mean (SD) & $48(12)$ \\
\hline \multicolumn{2}{|l|}{ Ethic origin } \\
\hline White British & $16(100)$ \\
\hline \multicolumn{2}{|l|}{ Education } \\
\hline No qualifications & $1(6)$ \\
\hline GCSE or O Level & $3(19)$ \\
\hline Post-GCSE or O Level & $4(25)$ \\
\hline Undergraduate degree & $4(25)$ \\
\hline Postgraduate qualification or higher & $4(25)$ \\
\hline \multicolumn{2}{|l|}{ Marital status } \\
\hline Married or cohabiting & $10(63)$ \\
\hline \multicolumn{2}{|l|}{ Number of children } \\
\hline Mean (SD) & $1(1)$ \\
\hline \multicolumn{2}{|l|}{ PHQ-9 (depression) score } \\
\hline Mean (SD) at baseline & $17(5)$ \\
\hline Mean (SD) at follow-up & $9(7)$ \\
\hline Follow-up PHQ-9 score $<10$ & $10(63)$ \\
\hline \multicolumn{2}{|l|}{ Adherence to Morita Therapy } \\
\hline Number of sessions attended (mean (SD)) & $7(4)$ \\
\hline Completed treatment & $7(44)$ \\
\hline Withdrew $\geq 5$ sessions & $4(25)$ \\
\hline Withdrew $<5$ sessions & $5(31)$ \\
\hline \multicolumn{2}{|l|}{ Morita therapist (of two available) } \\
\hline Therapist 01 & $8(50)$ \\
\hline \multicolumn{2}{|l|}{ Secondary SCID diagnoses (at baseline) } \\
\hline Any anxiety disorder & $10(63)$ \\
\hline Generalised anxiety disorder & $7(44)$ \\
\hline Panic disorder with agoraphobia & $4(25)$ \\
\hline Panic disorder without agoraphobia & $4(25)$ \\
\hline Social phobia & $2(13)$ \\
\hline Post-traumatic stress disorder & 1 (6) \\
\hline Obsessive compulsive disorder & $1(6)$ \\
\hline
\end{tabular}

Previous psychotherapy/counselling (at least one course of)

\begin{tabular}{lc} 
Any psychotherapy (not including counselling) & $12(75)$ \\
\hline Cognitive behavioural therapy & $10(63)$ \\
\hline Mindfulness-based cognitive therapy & $4(25)$ \\
Eye movement desensitisation and reprocessing & $1(6)$ \\
Other psychotherapy & $4(25)$ \\
Counselling & $8(50)$ \\
\hline
\end{tabular}

Percentages may not always total 100 due to rounding.

GCSE, General Certificate of Secondary Education; PHQ-9, Patient Health

Questionnaire 9; SCID, Structured Clinical Interview for DSM-V.

and understanding MT as a process; (4) facilitating the process: (overcoming) challenges and barriers and (5) the value and impact of MT. Each theme encompassed a number of constituent themes (figure 1). These themes were developed to explore and explain the relationships between the constituent themes and the acceptability of MT, within a model of how MT was experienced by different participants.

During analysis, it was clear that participants' views comprised different categories which linked to acceptability. Particularly salient was the sense that participants' expectations and understandings either facilitated or hindered their engagement with MT. To capture this, the first three themes essentially convey different typologies of participants' approaches towards and experiences of treatment: theme 1 contrasts themes 2 and 3 , with participants' accounts generally falling within either theme 1 or themes 2 and 3. Thus, participants who brought expectations of treatment which were inconsistent with MT generally misunderstood the approach and considered it to be unacceptable (theme 1), with a failure to either identify with the MT principles or understand treatment as a process to progress through. In contrast, those whose prior expectations and experiences facilitated their identification with the MT principles (theme 2) typically engaged with the approach from the offset, with their overwhelmingly positive experiences of treatment tied to their understanding of MT as a process (theme 3) and leading to positive accounts of the value and impact of MT (theme 5).

Theme 4 describes the difficulties participants experienced engaging with therapy on a practical rather than conceptual level. While whether such difficulties amounted to barriers to continuing treatment was often moderated by themes 1-3 (participants with incompatible expectations and understandings of treatment were less likely to tolerate such difficulties), this theme also captures how for some participants the principles of MT may be acceptable (ie, they identify with the principles as per theme 2) while the process of treatment is not. Thus, the relationship between this theme and the preceding themes highlights a key thread throughout participants' accounts and this model of MT: the distinction between MT in principle and practice. Overall, while an ability to identify with the principles manifested as highly important in seemingly priming participants for MT, the challenges of translating these principles into a process which is feasible to engage with (as per theme 4) further shaped acceptability.

Figure 1 illustrates the relationships between themes and how these shape an overall picture of engagement with, and acceptability and impact of, MT. This model is not intended to provide a representative account of acceptability across the themes: theme 1 , in which the most negative views are described, is dedicated to the accounts of a minority of participants who discontinued treatment and were purposively sampled to explore any issues with acceptability.

Participants' trial ID numbers are included in brackets after quotes. 


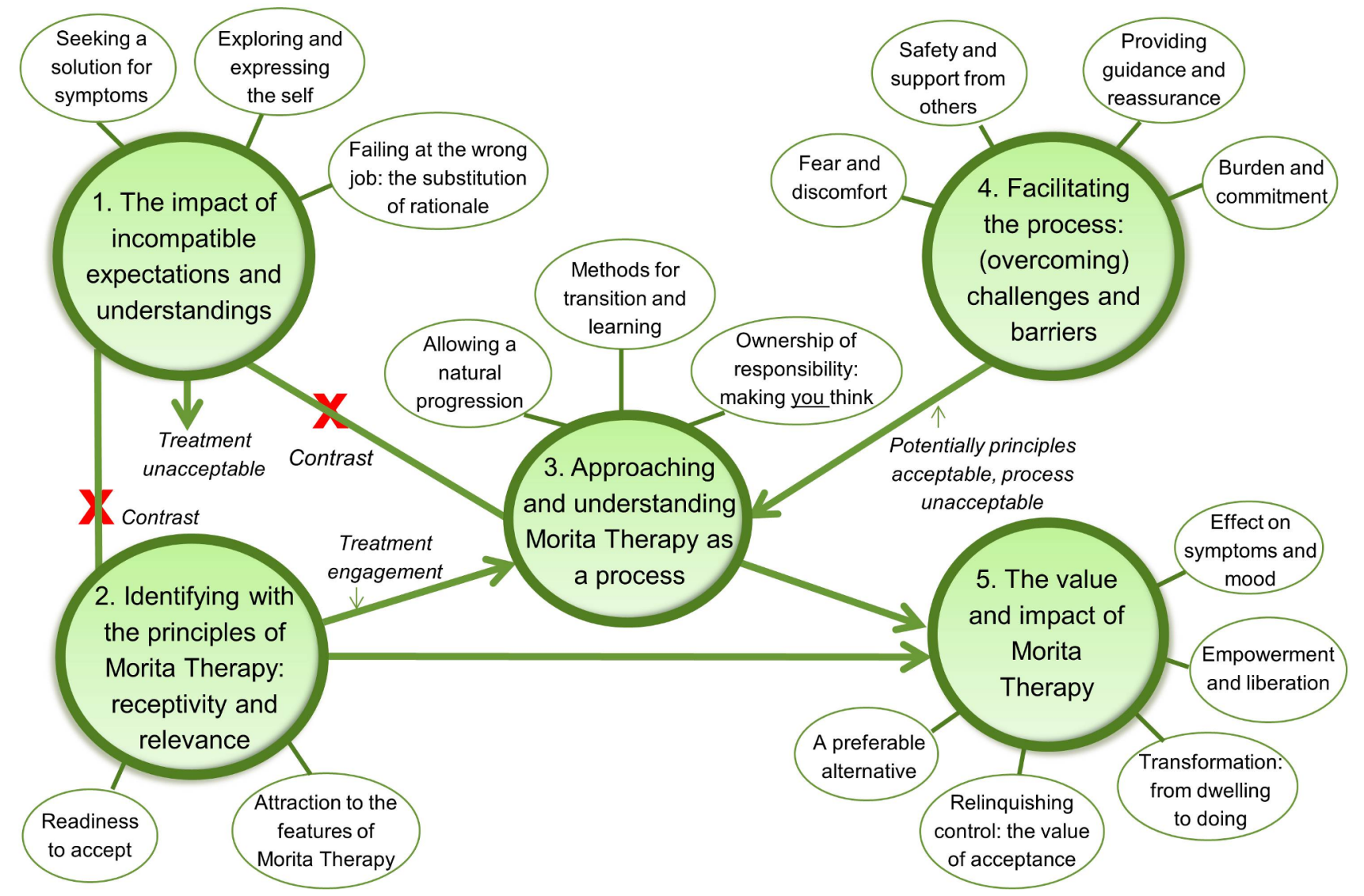

Figure 1 Thematic map.

Theme 1: the impact of incompatible expectations and understandings

Some participants demonstrated discrepancies between MT and their expectations of treatment; expectations which focused on seeking a solution for symptoms (constituent theme (a)) or exploring and expressing the self (constituent theme (b)). Participants also indicated how such expectations can feed the construction of rationales for treatment components which are inconsistent with MT (constituent theme (c)). As such, MT fails to achieve its assigned purpose, and/or fails to provide participants with the approach they seek. This theme contrasts with others: participants' accounts typically fall either within theme 1 or themes 2, 3 and 5 .

\section{(a)Seeking a solution for symptoms}

Several participants expressed a desire for therapy to provide a cure, answers or techniques to remove symptoms. Accordingly, participants resisted the underlying premise of MT to allow both pleasant and unpleasant internal states as natural and inevitable: their goal was to eliminate the unpleasant. These participants typically appeared to view the therapist as a holder of expert knowledge and abilities: someone who should 'fix' them or impart powerful techniques, with a sense of handing responsibility for both improvement and understanding therapy over to the therapist.
It's like a computer; you would replace the chip, why can't you do it in your head? It would just make you feel better... Why can't I be happy all the time instead of having one day good, one bad! (MT28)

\section{(b)Exploring and expressing the self}

Several participants expressed hopes that treatment would provide in-depth exploration, analysis and discussion of their difficulties.

I was hoping it was like a situation where I could open myself up... analysing why, you know, how I'm feeling...or why you feel bad. (MT61)

These expectations shaped views of MT, particularly Fumon (therapists' inattention to symptoms), with a sense that this somewhat stifled participants' self-expression and desire for someone to talk to and understand them. Thus, participants seeking a more exploratory and analytical approach felt somewhat 'shut down' (MT54) by and disappointed in MT.

(c)Failing at the wrong job: the substitution of rationale

For participants whose accounts fall within the constituent themes above, their expectations often shaped misunderstandings of the purpose of MT. In particular, participants typically substituted the rationale for rest (which is, primarily, to experience the natural ebb and 
flow of internal states) with one more consistent with their preconceptions. For example, a participant who sought a cure considered rest unable to help them 'conquer' their depression (MT51). Others considered rest an opportunity to have a relaxing 'break' from symptoms. Alternatively, a participant whose expectations focused on in-depth self-analysis, potentially on 'an unconscious level', had the following recollections of rest:

They said that we were gonna analyse your sleeping thing and arrange for you to sleep for a certain time... Actually planning something like that was really like, well, 'this isn't gonna work'. (MT61)

Thus, participants were assigning a MT incongruent purpose to rest; the achievement of which rest was not intended or able to fulfil. In turn, participants expressed a sense of both themselves and the therapy having 'failed' (MT19).

\section{Theme 2: Identifying with the principles of MT: receptivity and relevance}

In contrast to theme 1, many participants described approaching MT with insights, experiences and expectations which facilitated their identification with the MT principles, such as the underlying premise of accepting unpleasant thoughts and emotions (constituent theme (a)) and/or particular treatment components (constituent theme (b)).

\section{(a) Readiness to accept}

In recalling what appealed to them about MT before treatment, many participants expressed a sense of readiness to accept symptoms as part of oneself and life:

What attracted me was...it was a way of getting back to nature and realising that it's a part of you and part of the human experience, and stop catastrophising everything. (MT63)

This 'readiness to accept' appeared to be facilitated by participants' prior experiences of and insights into the nature of their difficulties: participants expressed understandings that symptoms could not be cured, they naturally come and go, and attempts to control them could worsen them. Thus, Moritian concepts of the 'vicious cycle' and the natural ebb and flow of internal states resonated strongly for such participants. These understandings were often shaped by previous treatment experience, typically $\mathrm{CBT}$ and counselling, which participants felt were too 'focused on your past and trying to stop you having these thoughts and feelings' (MT50) with potential to 'feed into' the vicious cycle.

[Morita Therapy] reinforced what I'd already hooked onto as a major problem for me... [CBT] was sort of feeding my need to fix myself... I came away from CBT going 'I've got to stop thinking these things, I've got to think differently' and you don't have that kind of control over your thoughts, I don't think. (MT45)
(b)Attraction to the features of MT

There was a sense from many participants that specific elements of MT 'grabbed' them from the offset and encouraged them to engage with the approach. Different features manifested as salient for different participants. Often, participants were attracted to the use of the natural world' (MT43), valuing both understanding human nature in relation to the natural world and a more literal engagement with nature. For others, key features included the focus on action-taking, understanding difficulties as reflections of underlying desires and working with the 'authentic self'.

The thought of somebody nurturing you and slowly trying to find what things you're looking for and what your values are and what little things you can go and do that are true to your authentic self... That's what I've been looking for, for the last twenty years! (MT50)

\section{Theme 3: approaching and understanding MT as a process}

Many participants understood the MT components as a part of a naturally unfolding progressive journey (constituent theme (a)) providing accumulative opportunities for learning and re-focusing attention (constituent theme (b)) and for owning responsibility for change (constituent theme (c)), as opposed to attempting to isolate each component as a potential technique for overcoming symptoms (as per theme 1).

\section{(a)Allowing a natural progression}

Many participants described MT as providing a natural and gentle progression. Within this, participants conveyed a sense of helpful balance in the process: the four-phased structure and therapist guidance were coupled with an individualised pace and lack of directive instructions. This enabled participants to gradually ‘build up' (MT16) themselves, their confidence and activity levels through achievable 'bite-sized' steps (MT43).

It was just this brilliant, gradual process, it sort of - the first stage broke me down, and then it was re-building me. (MT55)

Some participants also suggested that the purpose of treatment naturally unfolded through engagement with it, and highlighted the importance of MT as an experiential, rather than purely intellectual, process.

I've been allowed to discover it, guided gently and then I had to discover it for myself. And I think if you find it for yourself, and aren't following lots of instructions, it's almost like nature teaches you... It's kind of hit me at a bit of a visceral level. (MT63)

\section{(b)Methods for transition and learning}

Many participants spoke of MT in terms of providing accumulative opportunities for learning about human nature and transitioning from engagement in the vicious 
cycle to an acceptance of symptoms and external focus of attention. Key to this was the incorporation of methods, such as rest, diaries and natural-world metaphors, for learning about the transient nature of emotions.

Being with your thoughts and then learning that thoughts come and go... You relate it to different seasons of the year, and storms come, but they pass, and the sea goes calm and all of those sorts of things, you realise that happens with you naturally as a human being. (MT50)

Participants also spoke of the phases and diaries as a means of highlighting and enabling action-taking, and of Fumon (therapists' inattention to symptoms) as valuable for reducing the vicious cycle.

What was good about what [therapist] was doing was they would go 'Stop' as soon as I started that conversation, 'You're now scratching the itch', you know, 'Your mind wants to fix it and we're gonna sit here and fix it for half an hour, and fixing it's the problem, right?'... I did go away from - after a couple of sessions, thinking 'What they're actually saying is I'm just wasting my time'. (MT45)

In contrast to theme 1, these participants did not judge the treatment components in terms of how successful they were as tools for managing symptoms, but how successful they were as methods for learning and transition. This accurate understanding of the purpose of MT appeared crucial to participants' willingness to tolerate challenging components (such as rest), and how successful participants considered therapy to be.

\section{(c)Ownership of responsibility: making you think}

Many participants described the value of MT, particularly therapists' diary comments, partially in terms of 'making them think'. There was a welcome sense that, rather than the therapist providing answers and imparting knowledge, they provided subtle cues which encouraged participants to take responsibility for their own learning and application of the MT principles.

With the use of the diary, it's just picking out the salient points that are making you think... Instead of saying 'you need to do this'... [My therapist] was allowing me to pick up on very subtle signals, so - in trying to do that for myself. (MT33)

Through this, participants spoke of their therapist facilitating a re-evaluation of themselves and their lives, and equipping them to proceed post-treatment with a sense of self-efficacy: a desire to "walk on my own with Morita in mind' (MT63).

\section{Theme 4: Facilitating the process: (overcoming) challenges and barriers}

Participants described some challenges concerning the more practical rather than conceptual elements of MT, including fear and discomfort (constituent theme (a)), needing support from others (constituent theme (b)), needing sufficient therapist guidance (constituent theme (c)) and the commitment of treatment (constituent theme (d)). This highlights a distinction throughout participants' accounts between identification with the MT principles and the feasibility of engaging with the MT process itself.

\section{(a)Fear and discomfort}

Participants described fear and discomfort they had experienced at times during therapy, predominantly in relation to rest. This typically connected to participants having avoided their thoughts and feelings for some time. Whether or not the challenges of rest were acceptable to participants, or developed into barriers to continuing therapy, was linked to participants' expectations and understandings of treatment: those who assigned incorrect purposes to rest (as per theme 1) were disinclined to tolerate it; those who understood rest as a means of learning (as per theme 3) tended to persevere with it, acknowledging its importance.

[Therapist] said 'Just go with it, let it all come out', 'cos before I tried my hardest to block it off. After 9 days I thought 'Oh heck!'... But yeah, as I say, it worked. It wasn't pleasant but when I got to the end of it I could see we'd done it...'cos I learned that you can get through it and come out the other side. (MT55)

\section{(b)Safety and support from others}

Participants spoke of the need to feel safe, supported and encouraged by their significant others during therapy, with some describing a lack of support from others as a major factor in their decision to discontinue therapy. For one participant, their lack of personal safety and support led them to discontinue therapy despite their eagerness to continue, indicating the importance of creating a safe space for rest and the need for a certain degree of stability in participants' lives to facilitate engagement with MT:

Because of my neighbour who was being threatening and harassing, I didn't feel safe to sit in that environment... and my Mum started drinking terribly badly, so that all got so bad I just thought 'I need to do something quickly with my life'. (MT50)

With this requirement for safety and support manifesting particularly in relation to rest, several participants indicated that including their partner in the therapy session in which rest was explained helped to meet this need.

\section{(c)Providing guidance and reassurance}

Participants recalled some discomfort with a lack of clear instruction and sufficient reassurance being given around treatment components, particularly diaries. 
One thing I struggled with was having just a blank canvas in the diary... I found it quite hard to understand exactly what was needed. (MT16)

Participants indicated that providing a more detailed 'framework' (MT17) for diary completion and assuring participants that there is no 'correct' way of doing this were potential ways of minimising such challenges. In the context of therapy sessions feeling somewhat intensive and information rich, participants also suggested that providing audio-recordings and handouts, and ensuring regular input from participants during sessions, could facilitate their engagement with MT.

\section{(d)Burden and commitment}

There was a sense from participants that MT required a large commitment. Some participants had difficulties attending treatment sessions, or struggled to find time for components such as rest and diary writing. For several participants, this time commitment among work and caring responsibilities were key factors in their decision to discontinue therapy. For participants with incompatible expectations and understandings of treatment (theme 1), the requirements of MT also at times created a sense of burden.

It was pressurising me into doing it, every day you had to remember certain things... Then more and more, every week there's a bit more added and that's when it became too much. (MT28)

In the absence of understanding MT as a process alongside the failure of treatment components to achieve their assigned (incorrect) purpose (such as reducing symptoms), these participants appeared to consider these components as somewhat devoid of meaning and simply extra things which they 'had to do', creating a sense of pressure to 'perform' (MT19) for a therapy which was not working for them.

\section{Theme 5: the value and impact of MT}

Many participants, excluding those whose accounts fell within theme 1 , described the value and impact of MT in terms of providing a preferable alternative to other therapies (constituent theme (a)), the value of acceptance (constituent theme (b)), transformation from dwelling to doing (constituent theme (c)), empowerment and liberation (constituent theme (d)) and effect on symptoms (constituent theme (e)).

\section{(a)A preferable alternative}

Participants often described MT as preferable to (mindfulness-based) CBT and counselling, typically contrasting the accepting and allowing stance of MT with the controlling and combative stance of CBT. Participants welcomed a move from 'thinking positively' towards 'accepting that not everything is positive' (MT43) and considered the naturalisation of unpleasant experiences 'less judgemental and conflicting' than CBT (MT17). While participants viewed MT as a "philosophy to take you through life' (MT50) in which patients are 'not seen as a bunch of symptoms' (MT15), they described (mindfulness-based) CBT as a 'tool-kit' approach/'sticking plaster' (MT43) or 'short-term fix' (MT63) in comparison. There was thus a sense that MT was a more 'holistic' (MT43), pervasive and potentially sustainable approach, which had made fundamental and instinctive changes to their perspective.

I have done other work in the past but this seems to have struck a chord of change within, not just a 'Right, this is a strategy'... That never, ever worked for me. It's something fundamentally, I hope, I feel very optimistic, has changed with my acceptance of these feelings. (MT63)

\section{(b)Relinquishing control: the value of acceptance}

The impact of MT often centred on participants' acceptance and allowance of both pleasant and unpleasant experiences, and a sense of relinquishing attempts to control the uncontrollable. Critical to this was how therapy had normalised unpleasant emotions, making them permissible and demonstrating that it is 'okay to be with' them (MT33). Often, participants extended this attitude of acceptance more broadly in terms of 'what will be will be' (MT33) and 'it's just how it is' (MT43) with regards to all aspects of life they felt unable to control.

I don't worry about things, not anymore... [Therapist] has taught me to let things go, there's nothing you can do to change anything, if it's going to happen it will happen, you've just got to go with it and take the rough with the smooth... realising that has helped me. (MT37)

More widely, participants noted the positive impact of the acceptance, normalisation and permissibility of difficulties on their self-image and relationships, noting decreases in shame and criticism of both self and others.

\section{(c)Transformation: from dwelling to doing}

Participants described shifts in their attention and behaviour from fixating on symptoms towards focusing outwardly on the external environment: 'you're doing things rather than just dwelling on them' (MT43). Accordingly, participants often described paying less attention to (fixing) symptoms, being 'more present' in the moment (MT43) and 'getting more involved' (MT33) with others, activity, nature and life.

It's about moving your focus away from what's going on inside to carrying on what's going on in the real world... my mind is completely outside of myself, I'm looking forward and I'm interested in what I'm doing and I'm taking full part in it, and to be honest I don't even think about anxiety. (MT45)

There was thus a sense of transition from participants' lives being dictated by their symptoms to being dictated by external factors, with action-taking now being 
motivated by the action itself rather than a desire to overcome symptoms.

\section{(d)Empowerment and liberation}

Running through participants' accounts of acceptance and transition was a sense of empowerment through freedom from former restrictions, fears, judgements and struggles. Participants indicated that they were no longer 'scared' of their symptoms (MT15/MT50) and that relinquishing attempts to 'fix' them induced a sense of relief and liberation of energy.

I accept that it's almost, um, honestly being able to stop trying to cure yourself and just, yeah, give up that struggle... It's a feeling of - a little bit of relief. (MT45)

Participants also felt empowered to take action through learning that they can do so with symptoms and, as such, felt able to tackle avoided activities such as driving and changing jobs. This empowerment was often expressed in terms of increased self-confidence, feeling better equipped to dictate and manage situations, and feeling 'a lot more in control' (MT16) in terms of increased autonomy over their lives. This appeared to have manifested through a redirection of efforts and altered ownership of responsibility: through accepting what cannot be controlled (internal states) and focusing on what can be controlled (behaviour).

\section{(e)Improvement in symptoms and mood}

When questioned as to whether MT had helped them with their difficulties, participants often stated 'a lot', and described improvements such as stopping antidepressant medication. However, participants' spontaneous accounts typically focused on their changed outlooks and behaviours, prioritising the adoption of MT principles, with symptom reduction considered secondary to this or described through the lens of acceptance. While many referred to (an acceptance of) some continued symptoms, they typically noted these were of reduced duration due to reductions in the vicious cycle and increased action-taking. Similarly, participants described more frequent pleasant experiences, and more thorough engagement with and enjoyment of these, given this time was no longer spent attempting to analyse and pre-empt unpleasant experiences.

My anxiety's gone, my depression's gone and I'm in a much better place. I had a bad little patch...but then a day later I was absolutely fine, so instead of being stuck in that cycle for weeks, it was only like a couple of days. (MT50)

\section{DISCUSSION}

This paper presents a model of how different participants viewed and experienced MT, highlighting three key findings. First, the link between acceptability and participants' expectations, understandings and receptivity to the MT principles was demonstrated, suggesting some participants may find MT too discordant with their treatment expectations to be acceptable. Thus, the first three themes capture different typologies whereby participants who held expectations and understandings of treatment which are incompatible with MT (theme 1), such as seeking a cure for symptoms, were contrasted with those who identify with the principles (theme 2), such as accepting the natural ebb and flow of internal states, and accurately understand the purpose of the treatment components as part of a progressive process (theme 3 ).

Second, the distinction between MT in principle and practice was highlighted: while the centrality of identification with MT on a conceptual level was stressed, the challenges of engaging with MT on a practical level also shaped acceptability (theme 4 ). These challenges centred on fear and discomfort around rest, and other practical factors which are relevant in many forms of psychological therapy ${ }^{56}$ such as needing safety and support from others, needing sufficient therapist guidance and reassurance, and the required commitment. Whether participants were willing to tolerate such challenges was often shaped by the degree to which they identified with the MT principles and understood the purpose of the treatment components.

Finally, MT was perceived as acceptable by many participants, who emphasised the value and impact of the approach (theme 5) in terms of the acceptance, normalisation and permissibility of difficulties; a sense of transformation from life being dictated by symptoms to being dictated by external factors; empowerment and liberation from former restrictions, fears, judgements and struggles; and decreases in symptoms as a by-product of such changes. For these participants, MT was often described as preferable to other treatments they had tried.

\section{Strengths and limitations}

This study is the first to explore the views of UK-based patients about MT. Our use of qualitative methods enabled us to develop a rich account of acceptability within a model of how MT was experienced and understood by different participants, incorporating both exploratory and explanatory insights.

Our sample size was constrained by the number of Morita Trial participants meeting our sampling criteria. Nonetheless, it was possible to explore the views of participants who, together, fulfilled all manifestations of our sampling criteria, and only additional participants who both completed and responded to treatment were not sampled for analysis. Purposively (and explicitly) selecting participants to achieve maximum variation along theoretically driven dimensions allowed us to explore the breadth of views, obtain key insights in relation to issues with acceptability and conduct in-depth (non-superficial) analysis which represents the views of all of those sampled..$^{58}$ As analysis continued to the point at which no new themes were emerging and both the breadth and 
depth of data were explored, we consider data saturation and sampling adequacy to have been achieved. ${ }^{44} 49$

We recognise potential limitations of this study. The analysis may not have readily reflected how participants' views changed over time; for some factors deemed important in acceptability, such as identifying with the principles, the reliance on post-treatment interviews posed some challenges: it was difficult to ascertain the extent to which such identification had been held early in treatment (as claimed by many participants) and thus shaped views on acceptability versus the extent to which it emerged from participants' engagement in treatment. Therefore, in the future, it may be informative to capture the views and values of participants before as well as after treatment, to assess the nature of this relationship in more depth.

Furthermore, although our sample was diverse in terms of age and gender, the distribution of the education levels is higher than that found in the general UK population. ${ }^{59}$ Although the education distribution broadly reflects that of our whole pilot trial sample, ${ }^{29}$ those MT participants with whom we could not conduct an interview $(n=6)$ had a lower distribution of education level than those in our sample. It is possible therefore that our results are not transferable to the UK population as a whole, and moreover that education level may impact on the perceived acceptability of MT: the approach may be more attractive to and/or more suitable for those of a higher education level. Therefore, in a large-scale trial, it will be important to measure education level as a potential moderator of response and acceptability in MT.

\section{Implications and future research}

Our findings indicate that MT was acceptable to, and beneficial for, many participants. In the context of our promising pilot trial data on treatment adherence and outcomes (reported elsewhere), we suggest that the views of a minority of participants who found MT less acceptable should not prevent us from proceeding to a large-scale trial of MT using the UK Morita Therapy outpatient protocol. However, our results do suggest that some minor modifications to our clinical protocol may enhance acceptability. In particular, the provision of increased therapist explanation and reassurance around diary completion may ease some of the discomfort participants described in relation to this. Other suggestions made by participants, such as providing handouts during therapy, may also be incorporated.

One key finding is the link between acceptability and participants' identification with the MT principles in light of their expectations and understandings of treatment. This may inform further amendments to our clinical protocol in an effort to both better manage participants' expectations and better explain the purpose of the treatment components (eg, re-terming 'rest', which has connotations of 'relaxation', as 'being with' may clarify its purpose). This finding is also consistent with previous research which highlights the role of patients' expectations of and preferences for treatment in moderating treatment acceptability, engagement and response, ${ }^{60-63}$ and is an early indication of who MT may be more or less acceptable to. Our mixed methods study, reported elsewhere, extends these findings by relating them to treatment adherence and outcomes, and in a future definitive trial we seek to incorporate these potential moderators into a process evaluation. ${ }^{64}$

Many participants' accounts specifically highlight the novelty and value of MT in comparison to other treatments, with participants appreciating the distinctive focus on allowing (as opposed to controlling) symptoms. These data support the potential of MT to offer patients a meaningfully distinct alternative to other NICE-recommended treatments for current depression, thereby facilitating genuine patient choice as enshrined in the forthcoming NICE guidelines for depression. ${ }^{65}$ While our pilot trial results ${ }^{29}$ suggest that MT may be equivalent in effectiveness to other psychological therapies, treatment effectiveness varies at the level of the individual ${ }^{66}{ }^{67}$ : thus, while some participants who did not consider MT to be acceptable may be better suited to one of the currently available NICE-recommended treatments for depression, ${ }^{9}$ others may find MT more beneficial. Indeed, given the number of our participants who had tried other treatments in the past, these findings provide some early and tentative insights into the possible value of MT for some patients who do not respond to NICE-recommended first-line psychological treatments. In the context of little evidence to guide the management of these patients ${ }^{68}$ and an absence of a specific and effective treatment pathway within the NICE guidelines, it makes sense to test treatments which offer patients, for whom establish treatments have failed, a qualitatively different approach towards mental health.

The views of participants about MT may be of interest to clinicians and researchers more broadly. Although many have suggested that features of MT such as rest, Fumon (inattention to symptoms) and the concept of allowing rather than controlling symptoms may require dilution for a Western population, ${ }^{23}{ }^{69-72}$ we have tested a version of MT which closely aligns with the original four-phased inpatient model $^{21}$ by incorporating all such features. The views of many participants who valued not only MT but these specific features suggest that MT may not require as much modification to achieve cultural accommodation in the West as many authors have previously deemed necessary, yet not empirically investigated. $.^{23} 72-77$

Acknowledgements We thank the University of Exeter Mood Disorders Centre AccEPT Clinic for supporting this trial.

Contributors DAR proposed the study; HVRS as chief investigator and study researcher designed the study with the involvement of DAR and JF; JF provided additional guidance and support in relation to the qualitative study; HVRS drafted the study protocol and materials and obtained National Health Service ethical approval and research and development governance assurance; HVRS and DAR developed the UK Morita Therapy outpatient protocol; DAR supervised the study therapists. HVRS, who had undergraduate and master's degrees in the field of psychology as well as 7 years of experience in mental health research, was 
responsible for project management, data collection and analysis. HVRS managed the Morita Trial and conducted baseline assessments as part of her $\mathrm{PhD}$ and therefore had prior contact with all interviewees. HVRS received both in-house and external training in qualitative interviewing and analysis. JF supervised and conducted data analysis with HVRS. HVRS drafted the manuscript. All other authors contributed to editing of the final manuscript. All authors read and approved the final manuscript.

Funding The first author (HVRS) had a PhD fellowship award from the University of Exeter Medical School; DAR and JF are also funded by the University of Exeter Medical School and DAR, as a National Institute for Health Research Senior Investigator, receives additional support from the UK National Institute for Health Research South West Peninsula Collaboration for Leadership in Applied Health Research and Care. The AccEPT Clinic is funded by the National Health Service Northern, Eastern and Western Devon Clinical Commissioning Group and hosted by the University of Exeter's Mood Disorders Centre. The Morita Trial was sponsored by the University of Exeter (contact details available on request). The sponsor and funding sources have had no role in the design of this study, nor during its execution, analyses, interpretation of data or submission of results.

Competing interests None declared.

Patient consent for publication Not required.

Provenance and peer review Not commissioned; externally peer reviewed.

Data sharing statement The datasets generated and/or analysed during the current study are available from the corresponding author on reasonable request.

Open access This is an open access article distributed in accordance with the Creative Commons Attribution Non Commercial (CC BY-NC 4.0) license, which permits others to distribute, remix, adapt, build upon this work non-commercially, and license their derivative works on different terms, provided the original work is properly cited, appropriate credit is given, any changes made indicated, and the use is non-commercial. See: http://creativecommons.org/licenses/by-nc/4.0/.

\section{REFERENCES}

1. Mcmanus $S$, Bebbington $P$, Jenkins $R$, et al. Mental health and wellbeing in England: Adult Psychiatric Morbidity Survey 2014. Leeds 2016:39-40. http://digital.nhs.uk/catalogue/PUB21748 [Accessed 18 Aug 2017].

2. Kessler RC, Berglund P, Demler O, et al. The epidemiology of major depressive disorder: results from the National Comorbidity Survey Replication (NCS-R). JAMA 2003;289:3095-105.

3. Kessler RC, Berglund P, Demler O, et al. Lifetime prevalence and age-of-onset distributions of DSM-IV disorders in the National Comorbidity Survey Replication. Arch Gen Psychiatry 2005;62:593-602.

4. Keller MB. Long-term treatment of recurrent and chronic depression. J Clin Psychiatry 2001;62(Supplement_24):3-5.

5. Andrews G, Henderson S, Hall W. Prevalence, comorbidity, disability and service utilisation. Overview of the Australian National Mental Health Survey. Br J Psychiatry 2001;178:145-53.

6. O'brien M, Singleton N, Bumpstead R, et al. Psychiatric morbidity among adults living in private households, 2000. London: The Stationery Office 2001.

7. Marcus M, Yasamy MT, Ommeren m VAN, et al. Depression: A global public health concern. WHO Department of Mental Health and Substance Abuse 2012;1:6-8.

8. Bloom D, Cafiero E, JANÉ-Llopis E, et al. The Global Economic Burden of Noncommunicable Diseases (World Economic Forum, Geneva), 2011.

9. National Institute for Health and Clinical Excellence (NICE). Depression in adults: recognition and management [online]. 2009. https://www.nice.org.uk/guidance/cg90/chapter/1-Guidance\#step-3persistent-subthreshold-depressive-symptoms-or-mild-to-moderatedepression-with-inadequate [Accessed 21 Feb 2017].

10. National Collaborating Centre for Mental Health. Generalised Anxiety Disorder in Adults: Management in Primary, Secondary and Community Care. Leicester, London: The British Psychological Society and the Royal College of Psychiatrists (NICE Clinical Guidelines, No. 113), 2011.

11. Rush AJ, Fava M, Wisniewski SR, et al. Sequenced treatment alternatives to relieve depression $\left(\mathrm{STAR}^{\star} \mathrm{D}\right)$ : rationale and design. Control Clin Trials 2004;25:119-42.

12. Community \& Mental Health Team. Improving Access to Psychological Therapies (IAPT). Executive Summary (May 2016). NHS DIGITAL (GOVERNMENT STATISTICAL SERVICE), 2016.
Available: https://www.google.co.uk/url?sa=t\&rct=j\&q=\&esrc=s\& source $=$ web\&cd $=2 \& c a d=r j a \& u a c t=8 \& v e d=0$ ahUKEwj0o_vjmuvWAh XMiRoKHYagBZAQFggtMAE\&url=https\%3A\%2F\%2Fdigital.nhs. uk\%2Fmedia\%2F29276\%2FImproving-Access-to-PsychologicalTherapies-Executive-Summary-May-2016\%2FAny\%2FIAPT-monthMay-2016-exec-sum\&usg=AOvVaw3PdUxE3Y7ZIf1NI6jFz6eF [Accessed 12 Feb 2017].

13. IAPT. IAPT three-year report. The first million patients. London: 2012. https://www.google.co.uk/url?sa=t\&rct=j\&q=\&esrc=s\&source= web\&cd $=1 \&$ cad $=$ rja\&uact $=8 \&$ ved $=0$ ahUKEwjVr7WuIPXWAhXIzRo KHcspCJEQFggoMAA\&url=https\%3A\%2F\%2Fwww.uea.ac.uk\% 2Fdocuments\%2F246046\%2F11919343\%2FIAPT\%2B3\%2Byear\% 2Breport.\%2BThe\%2Bfirst\%2Bmillion\%2Bpatiets.pdf\%2F0e0469ff0884-4203-99de-4b61601e69dd\&usg=AOvVaw1NhSugavF4mlvy 9cRizLwq [Accessed 01 Aug 2017].

14. Amick HR, Gartlehner G, Gaynes BN, et al. Comparative benefits and harms of second generation antidepressants and cognitive behavioral therapies in initial treatment of major depressive disorder: systematic review and meta-analysis. BMJ 2015;351:h6019.

15. Depression Guideline Panel. Clinical practice guideline. Number 5. Depression in primary care. Rockville, MD: U.S. Department of Health and Human Services, Public Health Service, Agency for Health Care Policy and Research, 1993.

16. DeRubeis RJ, Hollon SD, Amsterdam JD, et al. Cognitive therapy vs medications in the treatment of moderate to severe depression. Arch Gen Psychiatry 2005;62:409-16.

17. Jarrett RB, Rush AJ. Short-term psychotherapy of depressive disorders: current status and future directions. Psychiatry 1994:57:115-32.

18. Luty SE, Carter JD, McKenzie JM, et al. Randomised controlled trial of interpersonal psychotherapy and cognitive-behavioural therapy for depression. Br J Psychiatry 2007:190:496-502.

19. Richards DA, Ekers D, McMillan D, et al. Cost and Outcome of Behavioural Activation versus Cognitive Behavioural Therapy for Depression (COBRA): a randomised, controlled, non-inferiority trial. Lancet 2016;388:871-80.

20. Westen D, Morrison K. A multidimensional meta-analysis of treatments for depression, panic, and generalized anxiety disorder: an empirical examination of the status of empirically supported therapies. J Consult Clin Psychol 2001;69:875-99.

21. Morita S, Kondo A, Levine P. Morita therapy and the true nature of anxiety-based disorders (Shinkeishitsu). New York, NY: State University of New York Press, 1998.

22. Kitanishi K. The Philosophical Background of Morita Therapy: Its Application to Therapy. In: Tseng WS, Chang SC, eds. NISHIZONO $M$. Honolulu, $\mathrm{HI}$ : Asian culture and psychotherapy University of Hawaii Press, 2005:169-85.

23. Ogawa B. Desire For Life: The Practitioner's Introduction to Morita Therapy. Indiana: Xlibris Corporation, 2013.

24. Nakamura K, Kitanishi K, Maruyama S, et al. Guidelines for practising outpatient morita therapy. Tokyo: Japanese Society for Morita Therapy, 2010.

25. Krech G. The Art of Taking Action: Lessons from Japanese Psychology. Monkton, VT: ToDo Institute, 2014.

26. Borglin $\mathrm{G}$. The value of mixed methods for researching complex interventions. In: Richards DA, Hallberg IR, eds. Complex Interventions in Health: An overview of research methods. Oxon, New York: Routledge, 2015:29-45.

27. Sugg HVR, Richards DA, Frost J. Optimising the acceptability and feasibility of novel complex interventions: an iterative, person-based approach to developing the UK Morita therapy outpatient protocol. Pilot Feasibility Stud 2017;3:37.

28. Sugg HV, Richards DA, Frost J. Morita therapy for depression and anxiety (Morita Trial): study protocol for a pilot randomised controlled trial. Trials 2016;17:161.

29. Sugg HVR, Richards DA, Frost J. Morita Therapy for depression (Morita Trial): a pilot randomised controlled trial. BMJ Open 2018;8:e021605.

30. Thabane L, Ma J, Chu R, et al. A tutorial on pilot studies: the what, why and how. BMC Med Res Methodol 2010;10:1.

31. Creswell JW, Plano Clark VL. Designing and conducting mixed methods research. Thousand Oaks: Sage, 2007.

32. Hill JJ, Kuyken W, Richards DA. Developing stepped care treatment for depression (STEPS): study protocol for a pilot randomised controlled trial. Trials 2014;15:452.

33. Richards DA, Hill JJ, Gask L, et al. Clinical effectiveness of collaborative care for depression in UK primary care (CADET): cluster randomised controlled trial. BMJ 2013;347:f4913.

34. Britten N. Qualitative research: qualitative interviews in medical research. BMJ 1995;311:251-3. 
35. Ritchie J, Lewis J, Nicholls CM, et al. Qualitative research practice: A guide for social science students and researchers. 2nd edn. Los Angeles, London, New Delhi, Singapore: Sage, 2013.

36. Taylor M. Interviewing. In: Holloway I, ed. Qualitative Research in Health Care. Maidenhead: Open University Press, 2011:29-55.

37. Marshall C, Rossman GB. Designing Qualitative Research. 5th edn. Thousand Oaks, CA: Sage, 2011.

38. Onwuegbuzie AJ, Leech NL. Sampling designs in qualitative research: Making the sampling process more public. Qual Rep 2007;12:238-54.

39. Miles MB, Huberman AM, Saldana J. Qualitative data analysis: $A$ methods sourcebook. 3rd edn. Los Angeles, London, New Delhi, Singapore: Sage, 2014.

40. Bryman A. Social research methods. 5th edn. Oxford: Oxford University Press, 2016.

41. Kroenke K, Spitzer RL, Williams JB. The PHQ-9: validity of a brief depression severity measure. J Gen Intern Med 2001;16:606-13.

42. Teddlie C, Yu F. Mixed methods sampling: A typology with examples. J Mix Methods Res 2007;1:77-100.

43. Gaskell G. Individual and Group Interviewing. In: Bauer M, Gaskell G, eds. Qualitative Researching with Text, Image, and Sound: $A$ Practical Handbook. London: Sage Publications, 2000:38-56.

44. Bowen GA. Naturalistic inquiry and the saturation concept: a research note. Qual Res 2008;8:137-52.

45. Marshall MN. Sampling for qualitative research. Fam Pract 1996;13:522-6.

46. Glaser B, Strauss G. The discovery of grounded theory: Strategies for qualitative work. New York: Aldine Publishing Company, 1967.

47. Mason M. Sample size and saturation in PhD studies using qualitative interviews. Forum Qual Soc Res 2010;11.

48. Morse JM. The significance of saturation. Thousand Oaks, CA: Sage, 1995;5:147-9.

49. Guest G, Bunce A, Johnson L. How many interviews are enough? An experiment with data saturation and variability. Field Methods 2006;18:59-82.

50. QSR International undated. NVIVO: THE \#1 Software for Qualitative Data Analysis.

51. Thorne S. Data analysis in qualitative research. Evid Based Nurs 2000;3:68-70.

52. Spencer L, Ritchie J, O'Connor W, et al. Analysis in practice. In: Ritchie J, Lewis J, MCNAUGHTON NICHOLLS C, eds. Qualitative research practice: A guide for social science students \& researchers. 2nd edn. London, Thousand Oaks, New Delhi, Singapore: Sage, 2014:295-346.

53. Miles MB, Huberman AM. Qualitative data analysis: An expanded sourcebook. Thousand Oaks, London, New Delhi: Sage, 1994.

54. Braun V, Clarke V. Using thematic analysis in psychology. Qual Res Psychol 2006;3:77-101.

55. Dingwall R, Murphy E, Watson P, et al. Catching goldfish: quality in qualitative research. J Health Serv Res Policy 1998;3:167-72.

56. Barnes M, Sherlock S, Thomas L, et al. No pain, no gain: depressed clients' experiences of cognitive behavioural therapy. $\mathrm{Br} J \mathrm{Clin}$ Psychol 2013;52:347-64.

57. Finning K, Richards DA, Moore L, et al. Cost and outcome of behavioural activation versus cognitive behavioural therapy for depression (COBRA): a qualitative process evaluation. BMJ Open 2017; 7:e014161.

58. Cleary M, Horsfall J, Hayter M. Data collection and sampling in qualitative research: does size matter? J Adv Nurs 2014;70:473-5.

59. Office For National Statistics 2012. 2011 Census: Key statistics for England and Wales, March 2011. Statistical Bulletin London.
60. Nilsson T, Svensson M, Sandell R, et al. Patients' experiences of change in cognitive-behavioral therapy and psychodynamic therapy: a qualitative comparative study. Psychotherapy Research 2007;17:553-66

61. Mcleod J. Qualitative research in counselling and psychotherapy. 2nd edn. Los Angeles, London, New Delhi, Singapore, Washington DC: Sage, 2011.

62. McLeod J. What do clients want from therapy? A practice-friendly review of research into client preferences. Eur J Psychother Couns 2012;14:19-32.

63. Swift JK, Callahan JL. The impact of client treatment preferences on outcome: a meta-analysis. J Clin Psychol 2009;65:368-81.

64. Moore G, Audrey S, Barker M, et al. Process evaluations of complex interventions: A summary of Medical Research Council guidance. In: Richards DA, Hallberg IR, eds. Complex Interventions in Health: An overview of research methods. Oxon, New York: Routledge, 2015:222-31.

65. National Institute For Health And Clinical Excellence (NICE). Depression in adults: treatment and management: Draft guidance consultation. [online]. Consultation. Available: https://www.nice.org. uk/guidance/indevelopment/gid-cgwave0725/consultation/htmlcontent [Accessed 01 Sep 2017].

66. Cuijpers $P$, Christensen $\mathrm{H}$. Are personalised treatments of adult depression finally within reach? Epidemiol Psychiatr Sci 2017;26:40-2.

67. Stiles WB, Shapiro DA, Elliott R. "Are all psychotherapies equivalent?". Am Psychol 1986;41:165-80.

68. Stimpson N, Agrawal N, Lewis G. Randomised controlled trials investigating pharmacological and psychological interventions for treatment-refractory depression. Systematic review. Br J Psychiatry 2002;181:284-94.

69. Kitanishi K, Mori A. Morita therapy: 1919 to 1995. Psychiatry Clin Neurosci 1995;49(5-6):245-54.

70. Kondo A. Translator's preface. In: Morita S, Kondo A, Levine P, eds. Morita therapy and the true nature of anxiety-based disorders (Shinkeishitsu). New York, NY: State University of New York Press, 1998:xi-xii.

71. Levine P. Editor's Introduction. In: Morita S, Kondo A, Levine P, eds. Morita therapy and the true nature of anxiety-based disorders (Shinkeishitsu). New York, NY: State University of New York Press, 199;8:xvii-xxvii.

72. Reynolds DK. Foreword. In: Kora T, ed. How to Live Well: Secrets of Using Neurosis. New York, NY: State University of New York Press, 1995:vii-ix.

73. Tanaka-Matsumi J. Cultural Differences and the Practice of Clinical Psychology in Japan and the United States: Cross-cultural Professional Experiences. In: Shimoyama H, ed. An International Comparison of Clinical Psychology in Practice: West Meets East. Tokyo: Kazama Shobo Inc, 2011:145-58.

74. Ishiyama I. Cross-cultural issues in introducing Morita therapy to the West. Journal of Morita Therapy 1994;5:57-60.

75. Ohara K. Creativity and experiential understanding in Morita Therapy. International Bulletin of Morita Therapy 1990;3:61-3.

76. Tseng W-S. Culture and Psychotherapy: Review and Practical Guidelines. Transcult Psychiatry 1999;36:131-79.

77. Reynolds DK. Flowing bridges, quiet waters: Japanese psychotherapies, Morita and Naikan. New York, NY: State University of New York Press, 1989. 